\title{
Behavior of the magnetic structures of the magnetic fluid film under tilted magnetic fields
}

\author{
H.C. Yang ${ }^{\mathrm{a}, *}$, I.J. Jang ${ }^{\mathrm{b}}$, H.E. Horng ${ }^{\mathrm{b}}$, J.M. Wu ${ }^{\mathrm{a}}$, Y.C. Chiou ${ }^{\mathrm{c}}$, Chin-Yih Hong ${ }^{\mathrm{c}}$ \\ ${ }^{a}$ Department of Physics, National Taiwan University, Taipei 106, Taiwan \\ ${ }^{\mathrm{b}}$ Department of Physics, National Taiwan Normal University, Taipei 117, Taiwan \\ ${ }^{\mathrm{c} D e p a r t m e n t ~ o f ~ M e c h a n i c a l ~ E n g i n e e r i n g, ~ D a-Y e h ~ U n i v e r s i t y, ~ C h a n g-H w a ~ 515, ~ T a i w a n ~}$
}

Received 20 May 1998; received in revised form 15 September 1998

\begin{abstract}
The patterns of the magnetic structure of the magnetic fluid thin film under tilted magnetic fields were taken to investigate the behavior of magnetic structures. The tilted angle $\theta$ is the angle between the direction of applied magnetic field and the normal line of the film. In our previous work, a nearly perfect ordered hexagonal structure in magnetic fluid thin film was obtained when a perpendicular magnetic field $(\theta=0)$ was applied. By increasing $\theta$, each column in the hexagonal structure gradually tilted to reveal a lattice of segments instead of dots on the pattern with an unchanged lattice distance $d$. As $\theta$ exceeding a critical value $\theta_{\mathrm{c}}$, these segments began to form longer periodic chains with an abrupt increasing of $d$. Hence, a transition from the magnetic hexagonal structure to a periodic chain structure at $\theta_{\mathrm{c}}$ existed for magnetic fluid thin film in which the $\theta_{c}$ decreases exponentially as the sweeping rate increases. (C) 1999 Elsevier Science B.V. All rights reserved.
\end{abstract}

Keywords: Magnetic fluid; Thin films; Ordered structures; Tilted magnetic field

\section{Introduction}

Pattern formation in magnetic fluid films subjected to external magnetic fields has recently attracted the interest of scientists due to their rich and diverse phenomena. The aggregated process and one-dimensional patterns in parallel magnetic fields were studied [1-3]. On the other hand, twodimensional ordered structures of magnetic particle columns were also observed in a magnetic hole of

* Corresponding author. Fax: 886-2-23639984.

E-mail address: hcyang@phys.ntu.edu.tw (H.C. Yang) ferrofluid [4], in the magnetic fluid emulsion [5], and in the magnetic fluid thin film [3]. However, the mechanisms of the transition between 2-D and 1-D patterns in the magnetic fluid thin film are not yet clear, and the behavior of the magnetic structures of the film under tilted magnetic fields have not yet been systematically studied as well.

In our past work, we discovered 2-D ordered structures in kerosene-based magnetic fluid film [3]. As a result, we had the magneto-chromatic effects observed under various perpendicular magnetic fields [6]. The relationship between the wavelength of a tunable grating using magnetic fluid thin film and the column distance in the film 
was studied and the grating model was established [7]. In order to obtain the most flexibility in the design of optical devices utilizing either hexagonal structures or periodic chains formed in magnetic fluid thin film, the study of ordered structures in magnetic fluid films under the tilted magnetic fields is necessary. Thus, we systematically investigated the behavior of the magnetic structures of the magnetic fluid thin film under tilted magnetic fields.

\section{Experimental details}

The magnetic fluid sample used in this study was a kerosene-based magnetic fluid prepared by the co-precipitation technique [3]. To form a magnetic fluid thin film, the sample was sealed in a $4 \mathrm{~cm} \times 1 \mathrm{~cm}$ rectangular glass cell with thickness of $6 \mu \mathrm{m}$. The tilted angle $\theta$, the angle between the direction of applied magnetic field and the normal line of the film, was adjustable. The resulting magnetic field generated by a solenoid coil was uniform with the deviation of the field strength in the whole region of the cell being less than $1 \%$. The distance $d$ between two segments or chains were obtained from photoimages which were taken by using a Leitz optical microscope and recorded in a personal computer through a CCD video camera.

\section{Results and discussion}

When a uniform perpendicular magnetic field $(\theta=0)$ was applied to the magnetic fluid film, a part of the magnetic particles aggregated to form magnetic columns. Moreover, an ordered and steady 2-D hexagonal structure was formed as the applied field exceeded a critical value of field strength $H_{\mathrm{h}}(\sim 80$ Oe for this sample) [3]. The final picture of the hexagonal structure under the perpendicular magnetic field $H=150 \mathrm{Oe}$ with its sweeping rate $\mathrm{d} H / \mathrm{d} t=5 \mathrm{Oe} / \mathrm{s}$ is shown in Fig. 1(a). By increasing $\theta$, it was found that each column in the hexagonal structure gradually tilted to reveal a lattice of segments instead of dots on the pattern with an unchanged lattice distance $d$, as shown in Fig. 1(b). As $\theta$ exceeding a critical value $\theta_{c}$, these segments began to form zig-zag-like short chains, shown in Fig. 1(c). It indicated that owing to the attractive magnetic force between two neighboring tilted columns the top of each tilted column began to connect with the bottom of its neighboring tilted column in the direction along the field. When $\theta$ was turned to a higher inclination, the magnetic particles re-distributed themselves in order to reach a new equilibrium state. In this phase, some of particle chains dissolved into the carrier and disappeared; and others absorbed neighboring chains and/or particles from the carrier and became coarse. Hence, a larger equilibrium distance between chains was observed, shown in Fig. 1(d)-(f). It is worthy to note that by a closer inspection of Fig. 1(c) and 1(d) chains were not perfectly aligned. This phenomenon may be attributed to the grain boundaries existed in the original hexagonal structure [3]. As $\theta$ approaches to $90^{\circ}$ (parallel magnetic field), the distance $d$ between chains was $6.0 \mu \mathrm{m}$ and the average diameter $D$ of the chain was found to be $2.1 \mu \mathrm{m}$. In a magnetic fluid system, the columns/chains should be aligned with the direction of the external field. Assuming that from the side view of the film to which a parallel magnetic field was applied, a hexagonal structure is formed, the required minimum thickness of the film is $7.3 \mu \mathrm{m}$ $(=\sqrt{3} d / 2+D)$. However, the cell thickness used in this study was $6 \mu \mathrm{m}$. Thus, the observed pattern under the parallel magnetic field can be identified as a mono-layer of periodic chains.

To study the mechanism of the magnetic structures under tilted magnetic fields, the distance between two columns/chains was measured. The columns (or tilted segments) distance $d$ in this work was the distance from one lattice point to the midpoint of its two neighboring lattice points and the chains distance $d$ was the distance between two neighboring chains. The relationship between columns/chains distance $d$ and $\theta$ with various sweeping rates is plotted in Fig. 2. The critical tilted angle $\theta_{\mathrm{c}}$ was then identified by observing the photoimages as the angle at which the chain formation is initiated. These curves indicate that a transition from the magnetic hexagonal structure to a periodic chain structure at $\theta_{\mathrm{c}}$ exists for the magnetic fluid thin film. The reason for the increase of the distance between chains from that between 
(a) $\theta=0$

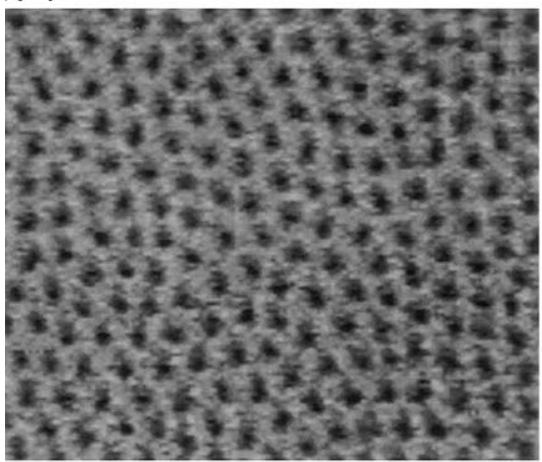

(c) $\theta=25$

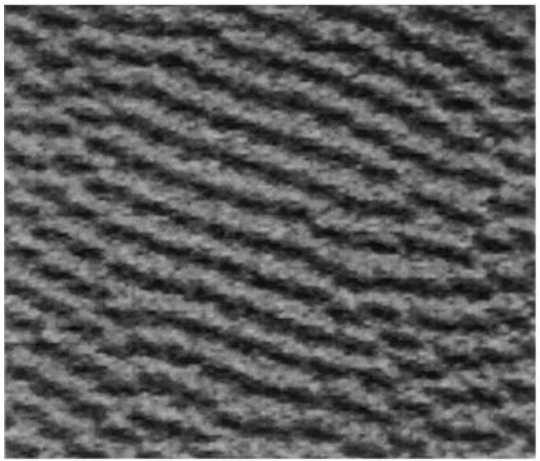

(e) $\theta=60$

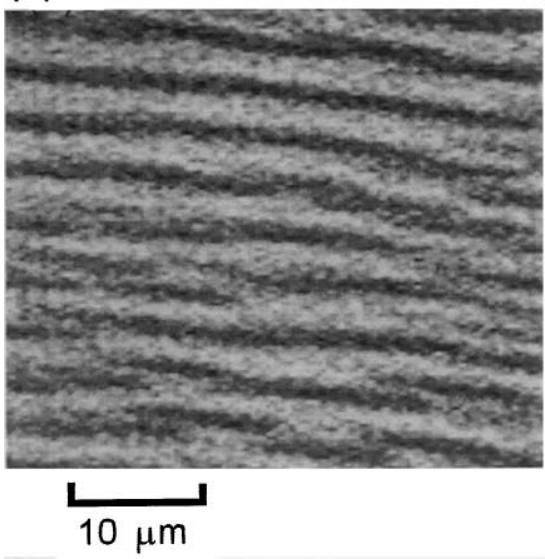

(b) $\theta=15$

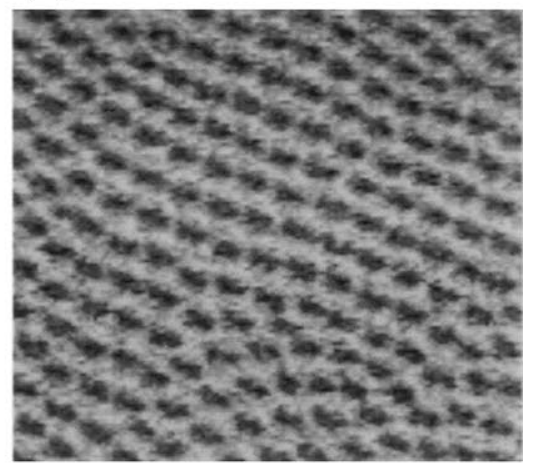

(d) $\theta=35$

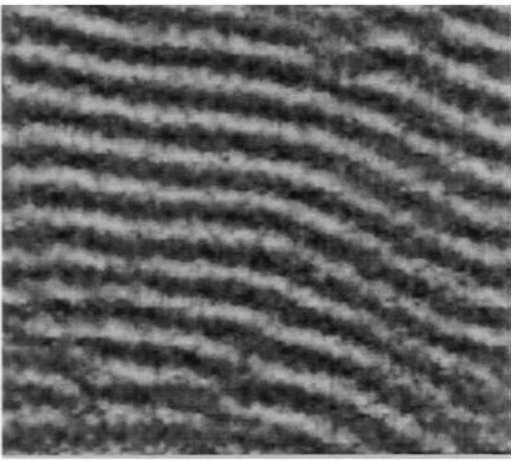

(f) $\theta=90$

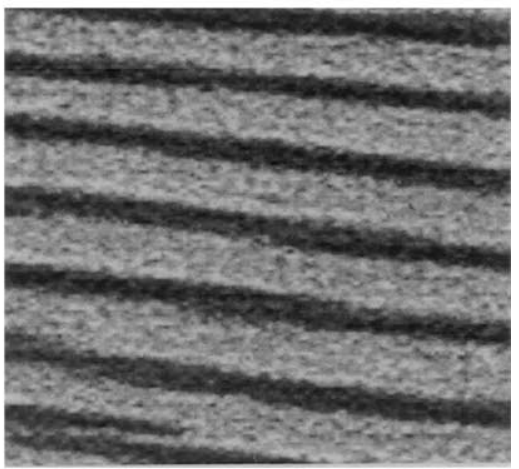

Fig. 1. Evolution of pattern formation of magnetic fluid thin film under tilted magnetic field with a fixed strength $H=150$ Oe. As the tilted angle $\theta$ increases from 0 , the structure evolves from the 2-D hexagonal structure to a periodic chain structure.

columns was caused by the increase of the magnetization in each chain compared to that in each column.
In our previous reports, the work points out that the sweeping rate of the perpendicular field affects the final column distances. The slower the sweeping 


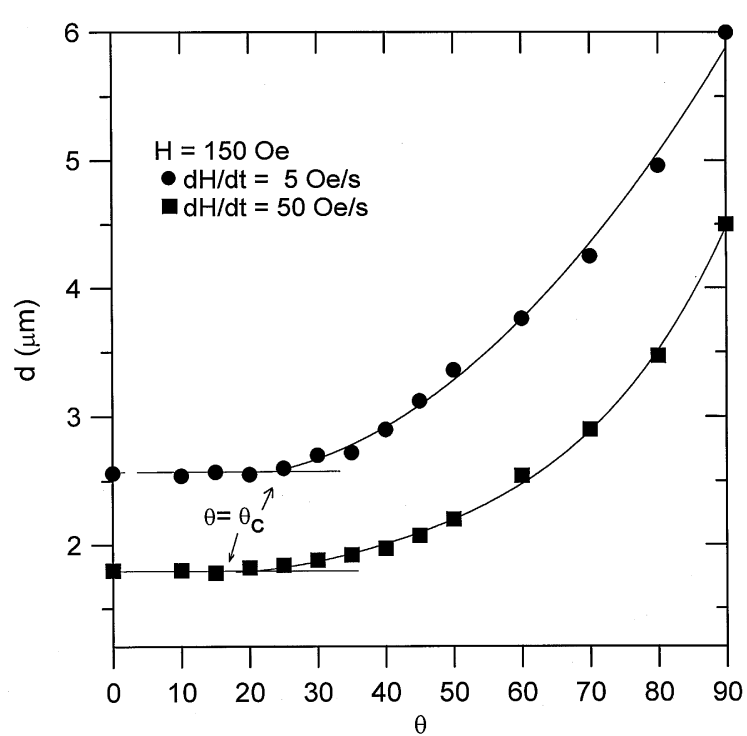

Fig. 2. The distance $d$ between columns/chains versus $\theta$ with various sweeping rate $\mathrm{d} H / \mathrm{d} t$.

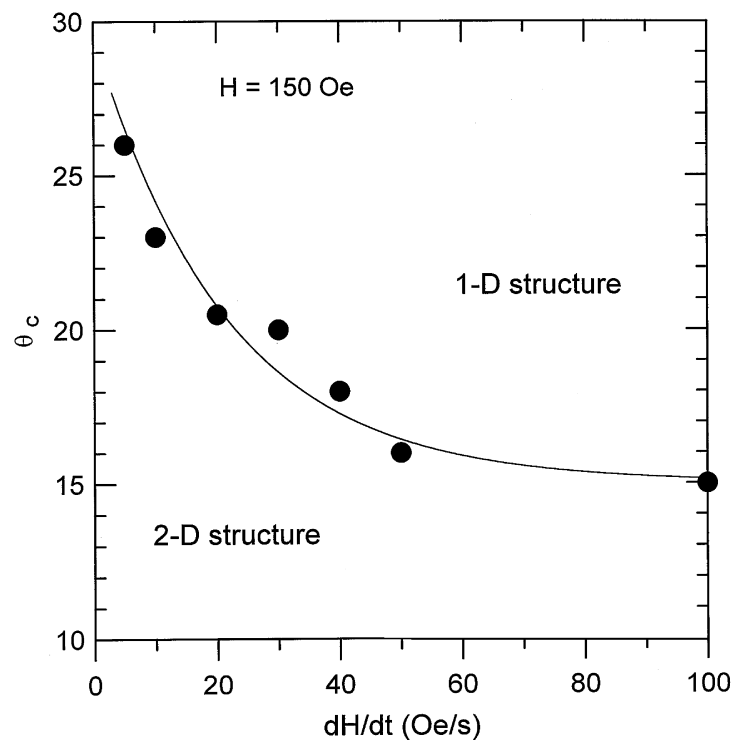

Fig. 3. The critical tilted angle $\theta_{\mathrm{c}}$ versus the sweeping rate $r(=\mathrm{d} H / \mathrm{d} t)$ of the magnetic field. The solid curve was the best fitting of an exponential function $\theta_{\mathrm{c}}=14.6\left(1+\mathrm{e}^{-0.046 r}\right)$.

rate of the field is, the farther away the columns become. The same behavior was also observed in this study. The 2-D hexagonal structure was observed at a lower tilted angle and slower sweeping rate. Contrarily, when the tilted angle or the sweeping rate increases, quasi-1D chains formed. Fig. 3 is a plot of $\theta_{\mathrm{c}}$ as a function of the sweeping rate $(\mathrm{d} H / \mathrm{d} t)$ under a fixed cell thickness. It indicated that $\theta_{\mathrm{c}}$ decreases exponentially versus sweeping rate $r=\mathrm{d} H / \mathrm{d} t$ with a best fitting $\theta_{\mathrm{c}}(r)=$ $14.6\left(1+\mathrm{e}^{-0.046 r}\right)$.

\section{Conclusions}

In summary, our experiment showed that a transition from the hexagonal structure to a structure of periodic chains exists in the magnetic fluid thin film subjected to a tilted magnetic field. And the critical value of the tilted angle $\theta_{c}$ is influenced by the sweeping rate $\mathrm{d} H / \mathrm{d} t$ of the magnetic field; and it decreases exponentially as the sweeping rate increases. At a small tilted angle $\theta$, it was found that each column in the hexagonal structure gradually tilts to reveal a lattice of segments instead of dots on the pattern with an unchanged lattice distance $d$. As $\theta$ exceeding $\theta_{\mathrm{c}}$, these segments began to form longer periodic chains with an increase of $d$. This was attributed to the increase of the magnetization in each chain.

\section{Acknowledgements}

This work was supported by the National Science Council under grant No. 87-2112-M-003001 and 87-2212-E-212-004.

\section{References}

[1] M. Fermigier, A.P. Gast, J. Colloid Interface Sci. 154 (1992) 522.

[2] D. Wirtz, M. Fermigier, Phys. Rev. Lett. 72 (1994) 2294.

[3] Chin-Yih Hong, I.J. Jang, H.E. Horng, C.J. Hsu, Y.D. Yao, H.C. Yang, J. Appl. Phys. 81 (1997) 4275.

[4] A.T. Skjeliorp, Phys. Rev. Lett. 51 (1983) 2306.

[5] J. Liu, E.M. Lawrence, A. Wu, M.L. Ivey, G.A. Flores, K. Javier, J. Bibette, J. Richard, Phys. Rev. Lett. 74 (1995) 2828.

[6] Herng-Er Horng, Chin-Yih Hong, Wai Bong Yeung, Hong-Chang Yang, Appl. Opt. 37 (1998) 2674.

[7] Chin-Yih Hong, H.E. Horng, I.J. Jang, J.M. Wu, S.L. Lee, Wai Bong Yeung, H.C. Yang, J. Appl. Phys. 83 (1998), in press. 